\section{DVT-Abrechnung leicht gemacht}

In einer gemeinsamen Arbeitsgruppe haben der Imaging-Spezialist Carestream Dental und die DKV Deutsche Krankenversicherung AG (DKV) beraten, wie sie das Abrechnungsprozedere für Digitale-Volumen-Tomografie-(DVT)-Aufnahmen vereinfachen können. Die Ergebnisse stellten die Akteure der DVT Abrechnungsinitiative am 10. Juli in Hamburg erstmals der Öffentlichkeit vor. Noch im September wird das Verfahren von der DKV umgesetzt.

Bei der Abrechnung von DVT-Aufnahmen besteht Handlungsbedarf. Denn sie ist sowohl für Behandler als auch für Versicherer oft problematisch. Häufig fehlen Informationen, wenn ein DVT beantragt oder abgerechnet wird. Die „DVT Abrechnungsinitiative“ hat nun einen Vorschlag erarbeitet, wie Indikation und Befund der 3D-Aufnahmen für die DKV einfach aufbereitet werden können, damit sie nachvollziehbar sind und ohne Verzögerung durch Rückfragen schneller bearbeitet werden können. Im Rahmen eines RoundTable-Meetings am 10. Juli in Hamburg präsentierten die Akteure der Initiative Frank Bartsch, Trade Marketing Manager bei Carestream Dental, Dr. Thomas Kiesel, Gesundheit bei der DKV und Priv.-Doz. Dr. Sigmar Kopp, Vertretern der dentalen Fachpresse die Ergebnisse der gemeinsamen Arbeit. Abteilungsleiter Kundenservice Leistung

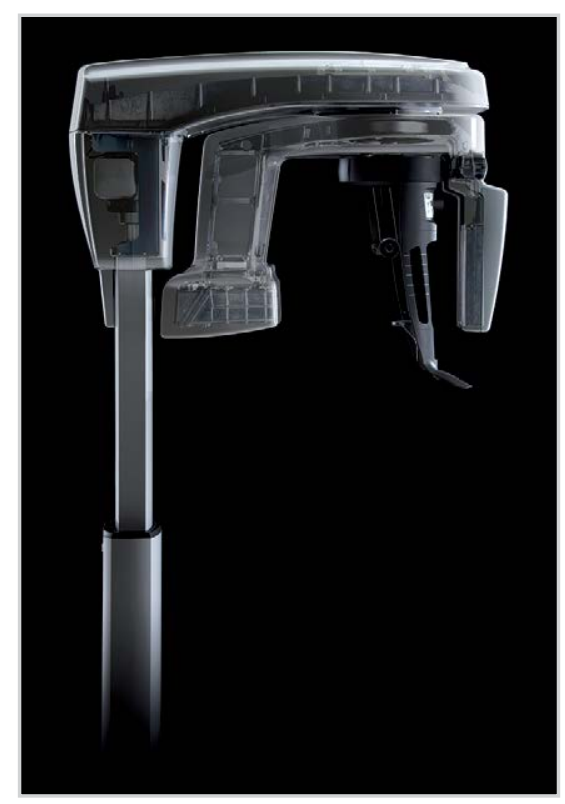

Komplizierte Abrechnung geht zu Lasten aller $\checkmark$

„Zum Teil erreichen uns mit den DVT-Erstattungsanträgen Daten-DVDs, die nur mit speziellen Programmen gelesen werden können“, berichtete Dr. Thomas Kie- 
sel, der auf 28 Jahre Berufserfahrung als Zahnarzt zurückblickt und seit 2002 für die DKV tätig ist. „Eine klare Struktur für die Zusammenstellung der relevanten Informationen kann den Vorgang erheblich beschleunigen“, verdeutlichte er die Notwendigkeit einer Veränderung im System. Ein großer Teil der Anträge für DVT-Aufnahmen seien nicht nachvollziehbar; viele Abrechnungen fehlerhaft. Wird eine Leistung verzögert oder mangels hinreichender Nachweise gar nicht erstattet, geht dies zu Lasten aller Beteiligten: „Der Zahnarzt steht im schlechtesten Fall im Verdacht, etwas abrechnen zu wollen, was nicht erstattungsfähig ist. Die Versicherung darf die Leistung nicht erstatten, weil der Fall nicht eindeutig geklärt ist. Und der Patient ist verärgert, weil er am Ende alles selbst zahlen muss“, beschreibt Dr. Kiesel die Folgen eines undurchsichtigen Verfahrens. Aus diesem Grund hat die DKV gemeinsam mit Carestream Dental die Initiative ergriffen.

\section{Abrechnungsempfehlung der BZÄK und der BÄK $\checkmark$}

Die Bundeszahnärztekammer weist für die Abrechnung von Anfertigung und Befundung einer DVT-Aufnahme die GOÄ Nummer 5370 aus. Die computergesteuerte Analyse einschließlich speziell nachfolgender 3D-Rekonstruktion soll nach der Zuschlagsziffer GOÄ 5377 berechnet werden. Die Bundesärztekammer legt die analoge Berechnung fest. Doch vielfach berechnen Zahnärzte anders. Auch fehlt es an einer einheitlichen Form für Informationsunterlagen zu DVT-Aufnahmen.

\section{Empfehlung der Arbeitsgruppe er- leichtert Indikatiosdarstellung $\nabla$}

Grundlage für eine Erstattung ist zunächst, dass eine medizinische Indikation für die Aufnahme vorliegt, wie Priv.-Doz. Dr. Sigmar Kopp ausführte: „Bevor es an die eigentliche DVT-Aufnahme und die spätere Erstattung geht, sind eine vernünftige Sichtung und eine sinnvolle Diagnostik notwendig. Anhand der Empfehlung der Arbeitsgruppe sollte der Behandler zunächst prüfen, ob die Indikation gerechtfertigt ist.“

Der in eigener Praxis in Rostock niedergelassene Behandler ist zusätzlich in Forschung und Lehre an der Universität Rostock tätig und Mitglied der Carestream Referenz Zahnärzte. Gemeinsam mit Dr. Kiesel hat er die s2k-Leitlinie Dentale digitale Volumentomographie für die Abrechnung von DVT-Aufnahmen unter diesen Gesichtspunkten geprüft. „Und zwar so, dass Graubereiche möglichst entfallen und eindeutige Situationen klar dargestellt werden können“, erklärte er. Herausgekommen ist dabei die Zusammenstellung der Indikationen, bei deren Vorliegen erfahrungsgemäß eine Erstattungsfähigkeit von der DKV anerkannt wird. Die Indikationsstellung ist in diesen Fällen regelmäßig nachvollziehbar und es erübrigen sich lästige Nachfragen von Seiten der DKV.

\section{Einfach zu erstellendes Formblatt beschleunigt Abrechnung}

Ein von der Arbeitsgruppe entwickeltes Muster-Formblatt erfasst den Fall entsprechend der definierten Indikation. „Es ist mit jedem DVT möglich, ein PDF zu erstellen, das die Parameter unseres Musterbeispiels sowie die DVT-Aufnahme enthält und dann bei der DKV eingereicht werden kann“, stellte Frank Bartsch diese wesentliche Vereinfachung bei der Erstellung der Informationen für die Abrechnung einer DVT-Aufnahme vor. „Alle Daten, die der Versicherer für die Abrechnung benötigt - also Indikation, Nummer, Kurzbefund und DVT-Aufnahme - sind in dem von uns erarbeiteten Formblatt enthalten. Die DVT-Indikationen sind so eindeutig nachvollziehbar und können von der DKV unproblematisch geprüft werden“, machte Dr. Kiesel die Vorteile des Formblattes für die DKV deutlich.
Auch für den Zahnarzt werden Dokumentation und Abrechnung so einfacher und übersichtlicher: „Die Ergebnisse der Initiative stellen für DVT-Anwender eine echte Erleichterung bei der Erfassung der Fälle dar. Gleichzeitig profitieren wir von einer besseren Transparenz der Erstattung“, so PD Dr. Kopp.

\section{Materialien stehen ab 15. Septem- ber zur Verfügung}

Während des Round-Table-Meetings kündigte Dr. Kiesel an, dass Behandler ab dem 15. September die von der Abrechnungsinitiative erarbeiteten Materialien nutzen können. Das Informationspaket enthält Abrechnungsempfehlungen, beispielhafte Musterfälle, ein Formblatt für die Information an die Kostenträger und eine Indikationsbeschreibung. Die Unterlagen stehen auf einer Webseite der DKV (www.dvt-abrechnung.de) zum Download bereit.

\section{Carestream Dental: Nah an den Bedürfnissen der Anwender \\ $\checkmark$}

Die „DVT Abrechnungsinitiative“ von Carestream und der DKV ist aus dem gemeinsamen Engagement im „Aktionsbündnis gesundes Implantat“, einem $\mathrm{Zu}$ sammenschluss verschiedener Organisationen, die sich für die Prophylaxe an Implantaten einsetzen, hervorgegangen. „Ob bei der Produktentwicklung, der Realisierung von erfolgreichen Projekten wie der Abrechnungsinitiative oder dem konstruktiven Austausch im Rahmen unserer Round Table Meetings mit Referenzzahnärzten: Es ist unser übergeordnetes Ziel, immer nah an den Bedürfnissen der Anwender zu sein“, beschreibt Frank Bartsch die Firmenphilosophie von Carestream Dental.

Nach einer Presseinformation der Carestream Health Deutschland $\mathbf{G m b H}$, Stuttgart www.carestreamdental.de europedental@carestream.com 